Quantum Information and Computation, Vol. 0, No. 0 (2003) 000-000

(C) Rinton Press

\title{
RELATIVE STATE MEASURES OF CORRELATIONS IN BIPARTITE QUANTUM SYSTEMS
}

\author{
PIERRE RUDOLFSSON \\ Department of Quantum Chemistry, Uppsala University, Box 518, \\ SE-751 20 Uppsala, Sweden \\ ERIK SJÖQVIST \\ Department of Quantum Chemistry, Uppsala University, Box 518, \\ SE-751 20 Uppsala, Sweden \\ Centre for Quantum Technologies, National University of Singapore, \\ 3 Science Drive 2, 117543 Singapore, Singapore
}

Received (received date)

Revised (revised date)

\begin{abstract}
Everett's concept of relative state can be viewed as a map that contains information about correlations between measurement outcomes on two quantum systems. We demonstrate how geometric properties of the relative state map can be used to develop operationally well-defined measures of the total correlation in bipartite quantum systems of arbitrary state space dimension. These measures are invariant under local unitary transformations and non-increasing under local operations. We show that some known correlation measures have a natural interpretation in terms of relative states.

Keywords: Correlations; relative states

Communicated by: to be filled by the Editorial
\end{abstract}

\section{Introduction}

Ever since the formulation of the EPR argument [1, the predicted correlations between outcomes of localized quantum tests have been considered a distinctive and important feature of quantum mechanics, with bearings on both interpretative issues [2, 3, 4] and possible applications [5, 6. There are several open problems related to correlations within the quantum mechanical framework. The most important one is probably the qualitative question whether a given $n$-partite state is separable or entangled, i.e., if the correlations between the subsystems can be prepared by local operations and classical communication, or if global unitary evolution (or a source of shared entanglement) is required. Related to this question is its quantitative counterpart: how much classical correlation and entanglement does a quantum state contain? This question has resulted in proposed measures of correlation and entanglement, which can be divided roughly into two categories: one that focuses on the violation of Bell-CHSH type inequalities [7, 8, and another that quantifies the ability of states to serve as a resource in some communication task, e.g., entanglement of formation [9] and distillable entanglement [10]. 
One of the contexts in which quantum correlations play a significant role is quantum measurement theory. The measurement process may be analyzed in terms of the correlations in a closed composite system consisting of a system of interest $S$ and an apparatus $A$. If we denote the basis states of $S$ and $A$ by $\left|s_{i}\right\rangle$ and $\left|a_{i}\right\rangle$, respectively, and if the former initially is in the superposition $\alpha\left|s_{0}\right\rangle+\beta\left|s_{1}\right\rangle$, then the measurement can be described in terms of a unitary evolution resulting in the transformation $\left(\alpha\left|s_{0}\right\rangle+\beta\left|s_{1}\right\rangle\right) \otimes\left|a_{0}\right\rangle \mapsto \alpha\left|s_{0}\right\rangle \otimes\left|a_{0}\right\rangle+\beta\left|s_{1}\right\rangle \otimes\left|a_{1}\right\rangle$. The entangled state of $S$ and $A$ corresponds to a superposition of the possible apparatus states, which seems to be in contradiction with the definite outcome presented by the apparatus. Everett's "relative state formulation of quantum mechanics" [11] provides a framework to deal with the $S+A$ correlation and circumvent the "measurement problem". It introduces a natural "if - then" perspective, equivalent to that of conditional probabilities: if we observe the outcome $a_{0}\left(a_{1}\right)$ then the state of $S$ is $s_{0}\left(s_{1}\right)$, and, according to Everett, that is all there is to know. Mathematically, this may be understood as a map from the space of apparatus states to that of the system of interest. In this framework, the entangled state is a representation of the relation between the possible outcomes in one measurement to those of another. This makes the relative state formalism and the notion of conditional states potentially useful to study correlations encoded in quantum states, as shown in e.g. the context of entanglement [12] and steerability [13.

The purpose of this paper is to develop operationally well-defined correlation measures for arbitrary bipartite states by using certain geometric properties of the corresponding relative state map. For pure states, these measures coincide with known entanglement measures such as concurrence hierarchies [14] and $I$ concurrence [15. We extend these pure state measures to arbitrary mixed bipartite systems for which we obtain measures that are invariant under local unitary transformations as well as non-increasing under local operations. On the other hand, these measures may increase under local operations and classical communication (LOCC), a feature that reflects the fact that they quantify the total correlation in mixed quantum states.

This paper is organized as follows. In the next section, we introduce the concept of relative states in Hilbert space and operator formalisms. While the former framework is restricted to pure bipartite states, the latter allows for an extension of the relative state description to arbitrary mixtures of bipartite states. In section 3, we demonstrate how to exploit the relative state concept to quantify correlations in bipartite quantum systems of arbitrary Hilbert space dimension. The correlation measures are illustrated in section 4. The paper ends with the conclusions.

\section{Relative states}

\subsection{Hilbert space formalism}

Let a bipartite system $S$ consisting of subsystems $A$ and $B$ be in a pure state $|\psi\rangle \in \mathcal{H}_{A} \otimes \mathcal{H}_{B}$. Let $\operatorname{dim} \mathcal{H}_{A}=\operatorname{dim} \mathcal{H}_{B}=d$ and $|\psi\rangle=\sum_{i j}^{d} \alpha_{i j}|i j\rangle$, where $\{|i j\rangle\}$ is a product basis of the joint state spact. ${ }^{a}$ Following Refs. [12, 16, 17, $|\psi\rangle$ defines the relative state map $L_{\psi}: \mathcal{H}_{A} \mapsto \mathcal{H}_{B}$. The relative state operator $L_{\psi}$, taking a state $|\varphi\rangle \in \mathcal{H}_{A}$ to a state $|\phi\rangle \in \mathcal{H}_{B}$ according to

$$
L_{\psi}|\varphi\rangle=\langle\varphi \mid \psi\rangle=|\phi\rangle,
$$

${ }^{a}$ If $d_{A}=\operatorname{dim} \mathcal{H}_{A}<d_{B}=\operatorname{dim} \mathcal{H}_{B}$ there exists a Schmidt decomposition with a maximum of $d_{A}$ components, hence the state is effectively a $d_{A} \otimes d_{A}$. 
may be viewed as a partial scalar product, i.e., $\langle\varphi \mid \psi\rangle \equiv\left(\langle\varphi| \otimes \hat{1}_{B}\right)|\psi\rangle$. The relative state operator can be expressed as $L_{\psi}=\hat{\alpha} T$, with $\hat{\alpha}=\sum_{i j} \alpha_{j i}|i\rangle\langle j|$ and $T$ denotes complex conjugation in the $\{|k\rangle\}$ basis. The map $L_{\psi}$ is anti-linear, i.e.,

$$
L_{\psi}\left(a\left|\varphi_{1}\right\rangle+b\left|\varphi_{2}\right\rangle\right)=a^{*} L_{\psi}\left|\varphi_{1}\right\rangle+b^{*} L_{\psi}\left|\varphi_{2}\right\rangle
$$

and becomes anti-unitary in the case of a maximally entangled $|\psi\rangle$. Furthermore, $L_{\psi}^{\dagger}: \mathcal{H}_{B} \mapsto$ $\mathcal{H}_{A}$ such that $L_{\psi} L_{\psi}^{\dagger}=\operatorname{Tr}_{A}|\psi\rangle\langle\psi|=\rho_{B}$ and $L_{\psi}^{\dagger} L_{\psi}=\operatorname{Tr}_{B}|\psi\rangle\langle\psi|=\rho_{A}$. The state $|\phi\rangle$ is subnormalized $\langle\phi \mid \phi\rangle=\left\langle\varphi\left|\rho_{A}\right| \varphi\right\rangle \leq 1$.

In the following, we refer to an argument $|\varphi\rangle$ of the relative state map as a hypo-state, which can be understood as an actual post-measurement state of one of the subsystems. The conditional state $|\phi\rangle$ we call re-state, short for a state relative to a hypo-state $|\varphi\rangle$.

The relative state map is a convenient way to express the fact that if Alice and Bob share the above pure bipartite state $|\psi\rangle$ and Alice chooses to measure an observable $Q$ with eigenstates $\left|\varphi_{k}\right\rangle$, then she can, when an outcome $k$ is obtained, predict the result of a specific projective measurement at Bob's site. If the shared state is entangled with $d$ non-zero Schmidt coefficients, there will be a one-to-one correspondence between states of $A$ and $B$, and each such pair is understood as a relative state.

\subsection{Operator formalism}

A more general approach to relative states can be developed in terms of linear maps of operators acting on Hilbert spaces $\mathcal{H}_{A}$ and $\mathcal{H}_{B}$. This framework allows for mixed hypo-states that may arise in non-projective measurements on one of the parties of bipartite states.

Denote by $\mathcal{B}(\mathcal{H})$ the space of Hermitian operators on $\mathcal{H}$. Let $\mathcal{S}(\mathcal{H})$ be the space of semipositive Hermitean operators with unit trace, and $\mathcal{S}^{\prime}(\mathcal{H})$ the non-negative cone of subnormalized density operators. A bipartite state $\varrho \in \mathcal{S}\left(\mathcal{H}_{A} \otimes \mathcal{H}_{B}\right)$ defines a map $\mathfrak{L}_{\varrho}: \mathcal{B}\left(\mathcal{H}_{A}\right) \mapsto \mathcal{B}\left(\mathcal{H}_{B}\right)$. If $Y \in \mathcal{B}\left(\mathcal{H}_{A}\right)$, then the map is given by

$$
\mathfrak{L}_{\varrho}(Y)=\operatorname{Tr}_{A}\left[\left(Y \otimes \hat{1}_{B}\right) \varrho\right] \in \mathcal{B}\left(\mathcal{H}_{B}\right) .
$$

For $Y$ being states $\tau$ of system $A$ (i.e., $\tau \in \mathcal{S}\left(\mathcal{H}_{A}\right)$ ), then $\mathfrak{L}_{\varrho}: \mathcal{S}\left(\mathcal{H}_{A}\right) \mapsto \mathcal{S}^{\prime}\left(\mathcal{H}_{B}\right)$ is the relative state map that takes hypo-states $\tau \geq 0$ on $\mathcal{H}_{A}$ to (subnormalized) re-states $\pi \geq 0$ on $\mathcal{H}_{B}$. The map $\mathfrak{L}_{\varrho}$ is linear in the space of density operators, i.e., if $a, a^{\prime}$ are real numbers and $\tau, \tau^{\prime} \geq 0$, then

$$
\mathfrak{L}_{\varrho}\left(a \tau+a^{\prime} \tau^{\prime}\right)=a \mathfrak{L}_{\varrho}(\tau)+a^{\prime} \mathfrak{L}_{\varrho}\left(\tau^{\prime}\right) .
$$

The norm of the re-state is the probability of finding the hypo-state in the global state.

A mixed hypo-state $\tau$ can be understood as the post-measurement state resulting from a (non-unique) set of projections obtained with certain probabilities. Alternatively, one may interpret the relative state map $\mathfrak{L}_{\varrho}: \mathcal{S}\left(\mathcal{H}_{A}\right) \mapsto \mathcal{S}^{\prime}\left(\mathcal{H}_{B}\right)$ in terms of an outcome $E=V \sqrt{\tau}$ $(\tau \geq 0$ and $V$ unitary) of a local generalized measurements on the $A$ system, resulting in the post-measurement state $\pi=\operatorname{Tr}_{A}\left(E \otimes \hat{1}_{B} \varrho E^{\dagger} \otimes \hat{1}_{B}\right)=\operatorname{Tr}_{A}\left(\tau \otimes \hat{1}_{B} \varrho\right)$ of the $B$ system.

We can represent density operators and observables as elements of a real vector space $\mathcal{V}$ and the relative state map can be expressed as a linear map of vectors. The corresponding vector elements can be interpreted as the expectation values of measured observables. Let 
$\left\{K_{k}^{A}\right\}_{k=1}^{d_{A}^{2}},\left\{K_{l}^{B}\right\}_{l=1}^{d_{B}^{2}}$ be bases of Hermitian operators on $\mathcal{H}_{A}$ and $\mathcal{H}_{B}$, satisfying the orthonormality conditions

$$
\operatorname{Tr}\left(K_{k}^{A} K_{k^{\prime}}^{A}\right)=\delta_{k k^{\prime}}, \operatorname{Tr}\left(K_{l}^{B} K_{l^{\prime}}^{B}\right)=\delta_{l l^{\prime}}
$$

A bipartite state can be expressed as

$$
\varrho=\sum_{k l} M_{k l} K_{k}^{A} \otimes K_{l}^{B}
$$

where

$$
M_{k l}=\operatorname{Tr}\left[K_{k}^{A} \otimes K_{l}^{B} \varrho\right] .
$$

The matrix $M$ is a representation of $\varrho$ with respect to the chosen basis. Local states $\tau, \pi$ are represented by real-valued vectors $\mathbf{a} \in \mathcal{V}\left(\mathcal{H}_{A}\right)$ and $\mathbf{b} \in \mathcal{V}\left(\mathcal{H}_{B}\right)$ with elements

$$
a_{k}=\operatorname{Tr}\left[K_{k}^{A} \tau\right], b_{l}=\operatorname{Tr}\left[K_{l}^{B} \pi\right] .
$$

We can express the map in Eq. (3) for $Y=\tau$ as

$$
\begin{aligned}
\tau=\sum_{j} a_{j} K_{j}^{A} \mapsto \pi & =\mathfrak{L}_{\varrho}\left(\sum_{j} a_{j} K_{j}^{A}\right) \\
& =\sum_{j k l} a_{j} M_{k l} \operatorname{Tr}\left[K_{j}^{A} K_{k}^{A}\right] K_{l}^{B}=\sum_{k l} a_{k} M_{k l} K_{l}^{B} .
\end{aligned}
$$

Hence, $b_{l}=\sum_{k} a_{k} M_{k l}$ or equivalently $\mathbf{b}=M^{\mathrm{T}} \mathbf{a}$. The relative state map is represented by

$$
M^{\mathrm{T}}: \mathcal{V}\left(\mathcal{H}_{A}\right) \mapsto \mathcal{V}\left(\mathcal{H}_{B}\right)
$$

and conversely

$$
M: \mathcal{V}\left(\mathcal{H}_{B}\right) \mapsto \mathcal{V}\left(\mathcal{H}_{A}\right)
$$

\section{Correlations}

A probability distribution $P(X, Y)$ over two random variables $X, Y$ taking values $x_{i}, y_{j}$ is correlated if $P(X, Y) \neq P(X) P(Y)$, where $P(X)$ and $P(Y)$ are the marginal distributions of $P(X, Y)$. The above condition can also be stated in terms of conditional probabilities: if there exist a pair $(i, j \neq i)$ such that

$$
P\left(X \mid Y=y_{i}\right) \neq P\left(X \mid Y=y_{j}\right),
$$

where $P\left(X \mid Y=y_{i}\right)$ denotes the probability distribution over $X$ given the outcome $Y=y_{i}$, then $P(X, Y)$ is correlated. The condition Eq. (12) states that a probability distribution is correlated if information about an outcome of $Y$ alters the prediction about the outcome of $X$ (and vice versa). Thus, a way to characterize the correlation in a probability distribution is to compare the set of conditional probabilities given an exhaustive set of mutually exclusive conditionals $\left\{y_{i}\right\}$, since the set of conditional probabilities contains information about how the 
random variables are correlated, i.e., which outcomes $x_{i}$ are correlated with which outcomes $y_{j}$.

A quantum state $\varrho \in \mathcal{S}\left(\mathcal{H}_{A} \otimes \mathcal{H}_{B}\right)$ is correlated if $\varrho \neq \rho_{A} \otimes \rho_{B}$, where $\rho_{A}$ and $\rho_{B}$ are the reduced states of the $A$ and $B$ subsystems, respectively. The relative state formalism allows us to employ Eq. (12) in a quantum context: a bipartite state $\varrho$ is correlated if there exists a pair of Hermitean operators $Y^{\prime} \neq Y$ such that

$$
\operatorname{Tr}_{A}\left(Y^{\prime} \otimes \hat{1}_{B} \varrho\right) \neq \lambda \operatorname{Tr}_{A}\left(Y \otimes \hat{1}_{B} \varrho\right)
$$

for any real number $\lambda$. Here, the conditional probabilities are replaced by relative states. The basic idea of the following analysis is to measure correlations in terms of how much the re-states differ for different choices of hypo-states. Specifically, the aim is to quantify correlations in terms of the difference of the conditional predictions contained in the re-states. In this way, we demonstrate how the geometrical properties of the relative state map can be used to develop correlation measures in arbitrary bipartite quantum systems.

\subsection{Pure state correlation measures}

Let us consider the bipartite pure product state $|\psi\rangle=\left|\psi_{A}\right\rangle \otimes\left|\psi_{B}\right\rangle$ and the corresponding relative state map $L_{\psi}$. For any hypo-state $|\varphi\rangle \in \mathcal{H}_{A}$, the re-state is

$$
|\phi\rangle=\langle\varphi \mid \psi\rangle=\left\langle\varphi \mid \psi_{A}\right\rangle\left|\psi_{B}\right\rangle,
$$

i.e., $L_{\psi}$ maps the whole Hilbert space $\mathcal{H}_{A}$ to the same ray in $\mathcal{H}_{B}$, that is, to the same state. This expresses the fact that for an uncorrelated state $\left|\psi_{A}\right\rangle \otimes\left|\psi_{B}\right\rangle$, a measurement outcome at site $A$ does not change the predictions about measurements at site $B$. Now, consider instead an entangled two-qubit state $|\psi\rangle \in \mathcal{H}_{A} \otimes \mathcal{H}_{B}$. If we choose two hypo-states $|\varphi\rangle,\left|\varphi^{\prime}\right\rangle$ such that the corresponding re-states $|\phi\rangle,\left|\phi^{\prime}\right\rangle$ are non-zero, then $|\varphi\rangle \neq\left|\varphi^{\prime}\right\rangle$ implies that $|\phi\rangle \neq z\left|\phi^{\prime}\right\rangle$, i.e., a measurement outcome at site $A$ does change the predictions regarding measurements at site $B$, as illustrated in Fig. 1.

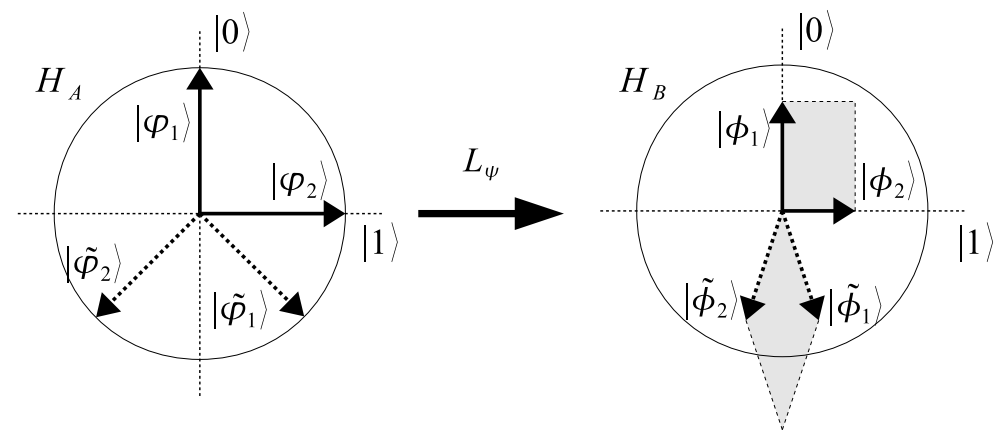

Fig. 1. An illustration of the correlation measure for a $2 \otimes 2$ system in pure state $|\psi\rangle=c_{0}|00\rangle+$ $c_{1}|11\rangle$. The picture shows the real planes in $\mathcal{H}_{A}$ and $\mathcal{H}_{B}$ spanned by the local Schmidt bases. Two different choices of orthonormal hypo-states in $\mathcal{H}_{A},\left|\varphi_{i}\right\rangle$ and $\left|\tilde{\varphi}_{i}\right\rangle$, maps to their respective re-states $\left|\phi_{i}\right\rangle$ and $\left|\tilde{\phi}_{i}\right\rangle$ in $\mathcal{H}_{B}$. The areas spanned by the restates are shown in gray.

By using these properties of the relative state map we may develop measures that quantify bipartite correlations. These measures are based upon geometric properties of the wedge 
product, $\wedge$ defined as follows. Let $\{|j\rangle\}_{j=1}^{d}$ be an orthonormal basis of a $d$ dimensional Hilbert space $\mathcal{H}$. Consider the vectors $\left|\xi_{i}\right\rangle=\sum_{j=1}^{d} \eta_{j}^{(i)}|j\rangle, i=1, \ldots, k \leq d$. We define the $k$-product of these vectors as

$$
\xi_{1} \wedge \cdots \wedge \xi_{k} \sim\left|\xi_{1}\right\rangle \wedge \cdots \wedge\left|\xi_{k}\right\rangle \equiv \sum_{1 \leq \mu_{1}<\ldots<\mu_{k} \leq d} \sum_{j_{1} \ldots j_{k}} \epsilon_{j_{1} \ldots j_{k}}^{\mu_{1} \ldots \mu_{k}} \eta_{j_{1}}^{(1)} \cdots \eta_{j_{k}}^{(k)}\left|\mu_{1} \ldots \mu_{k}\right\rangle,
$$

where $\epsilon_{j_{1} \ldots j_{k}}^{\mu_{1} \ldots \mu_{k}}$ is the Levi-Civita tensor, defined as $\epsilon_{j_{1} \ldots j_{k}}^{\mu_{1} \ldots \mu_{k}}=+1(-1)$ if $j_{1} \ldots j_{k}$ is an even (odd) permutation of $\mu_{1} \ldots \mu_{k}$ and zero otherwise. Note in particular that the $k$-product vanishes if $\xi_{i}$ are linearly dependent. $\xi_{1} \wedge \cdots \wedge \xi_{k}$ is an element of the exterior space $\Omega^{k}(\mathcal{H})$ with norm

$$
\left|\xi_{1} \wedge \cdots \wedge \xi_{k}\right|^{2} \equiv \sum_{1 \leq \mu_{1}<\ldots<\mu_{k} \leq d}\left|\sum_{j_{1} \ldots j_{k}} \epsilon_{j_{1} \ldots j_{k}}^{\mu_{1} \ldots \mu_{k}} \eta_{j_{1}}^{(1)} \cdots \eta_{j_{k}}^{(k)}\right|^{2} .
$$

For a set of vectors $\left\{\mathbf{v}_{i}\right\}$ in a real three dimensional vector space $\mathcal{V}^{3}$, the two-fold wedge product $\mathbf{v}_{i j}=\mathbf{v}_{i} \wedge \mathbf{v}_{j} \in \Omega^{2}\left(\mathcal{V}^{3}\right)$ can be identified with the directed surface element spanned by the two vectors, with area $\left|\mathbf{v}_{i j}\right|$. Correspondingly, the three-fold wedge product $\mathbf{v}_{i j k}=$ $\mathbf{v}_{i} \wedge \mathbf{v}_{j} \wedge \mathbf{v}_{k} \in \Omega^{3}\left(\mathcal{V}^{3}\right)$ represents a directed volume element, with volume $\left|\mathbf{v}_{i j k}\right|$ (see Fig. 2 for an illustration). This geometric intuition carries over to complex higher dimensional spaces; the $k$-fold wedge product $\xi_{i_{1} \ldots i_{k}}=\xi_{i_{1}} \wedge \cdots \wedge \xi_{i_{k}}$ can be seen as the oriented $k$-dimensional rhomboid spanned by the vectors, with $k$-volume $\left|\xi_{i_{1} \ldots i_{k}}\right|$.

Given a general bipartite system prepared in the pure state $|\psi\rangle \in \mathcal{H}_{A} \otimes \mathcal{H}_{B}$, where we assume that $\operatorname{dim} \mathcal{H}_{A}=\operatorname{dim} \mathcal{H}_{B}=d$, a set of hypo-states $\left\{\left|\varphi_{i}\right\rangle\right\}_{i=1}^{d},\left|\varphi_{i}\right\rangle \in \mathcal{H}_{A}$, is chosen such that $\operatorname{Sp}\left\{\left|\varphi_{i}\right\rangle\right\} \cong \mathcal{H}_{A}$. We obtain a set of re-states $\left\{\left|\phi_{i}\right\rangle\right\}_{i=1}^{d},\left|\phi_{i}\right\rangle \in \mathcal{H}_{B}$, via $\left|\phi_{i}\right\rangle=$ $L_{\psi}\left|\varphi_{i}\right\rangle=\left\langle\varphi_{i} \mid \psi\right\rangle$. Our basic measure of correlation with respect to any $k$-tuple of hypo-states $\left\{\left|\varphi_{i_{1}}\right\rangle, \ldots,\left|\varphi_{i_{k}}\right\rangle\right\} \subseteq\left\{\left|\varphi_{i}\right\rangle\right\}_{i=1}^{d}$ is given by

$$
\lambda_{i_{1} \ldots i_{k}}=\frac{\left|\phi_{i_{1}} \wedge \cdots \wedge \phi_{i_{k}}\right|}{\left|\varphi_{i_{1}} \wedge \cdots \wedge \varphi_{i_{k}}\right|}
$$

We may interpret $\lambda_{i_{1} \ldots i_{k}}$ as follows. Each $k$-tuple $\left\{\left|\varphi_{i_{1}}\right\rangle, \ldots,\left|\varphi_{i_{k}}\right\rangle\right\} \subseteq\left\{\left|\varphi_{i}\right\rangle\right\}_{i=1}^{d}$ of hypo-states is a basis of a $k$-dimensional subspace $\mathcal{H}_{A}^{i_{1} \ldots i_{k}} \subseteq \mathcal{H}_{A}$, and we will call $\lambda_{i_{1} \ldots i_{k}}$ a measure of the $k$-level correlation between that subspace and subsystem $B$.

To make this notion clearer, consider a $3 \otimes 3$ system in the state $|\psi\rangle=\sum_{i=1}^{3} \sqrt{p_{i}}|i i\rangle$, and a choice of hypo-states as $\left|\varphi_{i}\right\rangle=|i\rangle$, with the corresponding re-states given by $\left|\phi_{i}\right\rangle=L_{\psi}\left|\varphi_{i}\right\rangle=$ $\sqrt{p_{i}}|i\rangle$. By Eq. (17), we have three quantities for the two-level correlations $\lambda_{i j}=\left|\phi_{i} \wedge \phi_{j}\right|=$ $\sqrt{p_{i} p_{j}}, i<j$, and one for the three-level correlation $\lambda_{123}=\left|\phi_{1} \wedge \phi_{2} \wedge \phi_{3}\right|=\sqrt{p_{1} p_{2} p_{3}}$, see Fig. 2b where a similar example with a different choice of hypo-states is illustrated. The quantity $\lambda_{13}=\sqrt{p_{1} p_{3}}$ quantifies the difference between the restates $\left|\phi_{1}\right\rangle,\left|\phi_{3}\right\rangle$, and hence corresponds to how much our predictions about measurements on system $B$ differs with the two post-measurement states $\left|\varphi_{1}\right\rangle,\left|\varphi_{3}\right\rangle$ of $A$, i.e., when the outcome corresponding to $\left|\phi_{2}\right\rangle$ is discarded. Equivalently, $\lambda_{13}$ measures the effective $2 \otimes 2$ entanglement in the state $\left|\psi^{\prime}\right\rangle=(|1\rangle\langle 1|+| 3\rangle\langle 3|) \otimes(|1\rangle\langle 1|+| 3\rangle\langle 3|)|\psi\rangle$ resulting from a projection onto the subspace $\mathcal{H}_{A}^{13} \otimes \mathcal{H}_{B}^{13}$. The three-level quantity $\lambda_{123}$ measures the volume spanned by the re-states, i.e., how much the predictions differ when all three post-measurement states $\left|\varphi_{i}\right\rangle$ are taken into 
account. On the other hand, if $p_{3}=0$, then $\lambda_{13}=\lambda_{23}=\lambda_{123}=0$, where $\lambda_{13}=\lambda_{23}=0$ reflects that $\left|\psi^{\prime}\right\rangle=\sqrt{p_{1}}|11\rangle$ is a product state (the subspace $\mathcal{H}_{A}^{13}$ is not correlated with $B$ ), and $\lambda_{123}=0$ means that there exist no correlations that is not two-level. As is shown in Fig. $2 v$, the linear dependence of the re-states tells us that the information in, e.g., $\left|\phi_{3}\right\rangle$, is already present in $\left|\phi_{1}\right\rangle,\left|\phi_{2}\right\rangle$. Note that the denominator of Eq. (17) can be viewed as a normalization factor quantifying how much the hypo-states differ in the first place.
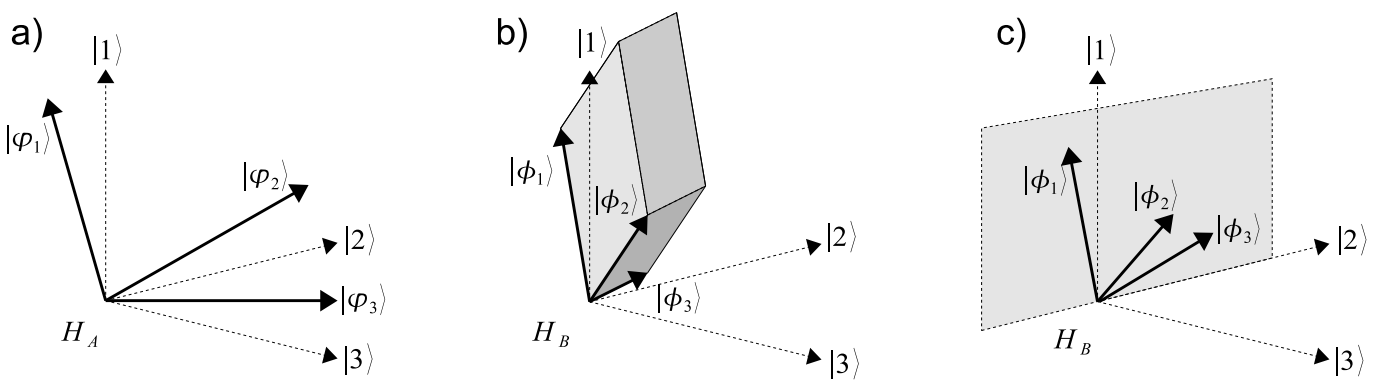

Fig. 2. An illustration of the correlation measures $\lambda_{i j}, \lambda_{123}$ for a $3 \times 3$ system in two pure states with different Schmidt-number, $|\psi\rangle=\sum_{k=1}^{3} c_{k}|k k\rangle$ and $|\widetilde{\psi}\rangle=\sum_{k=1}^{2} \widetilde{c}_{k}|k k\rangle$. In a) a choice of hypostates $\left|\varphi_{i}\right\rangle \in \mathcal{H}_{A}$ is depicted, and b) shows the re-states $L_{\psi}\left|\varphi_{i}\right\rangle=\left|\phi_{i}\right\rangle \in \mathcal{H}_{B}$, which span the volume $\lambda_{3}=\left|\phi_{1} \wedge \phi_{2} \wedge \phi_{3}\right|$ taken as the measure of three-level correlations. The areas of the faces of the rhomboid are given by $\lambda_{12}=\left|\phi_{1} \wedge \phi_{2}\right|, \lambda_{13}=\left|\phi_{1} \wedge \phi_{3}\right|$ and $\lambda_{23}=\left|\phi_{2} \wedge \phi_{3}\right|$, and they are measures of the two-level correlations bewteen the respective two-dimensional subspaces. In c) the re-states $L_{\widetilde{\psi}}\left|\varphi_{1}\right\rangle=\left|\widetilde{\phi}_{i}\right\rangle$ are shown, which lies in the subspace (shown in gray) spanned by the Schmidt vectors $|1\rangle,|2\rangle$. Consequently, for $|\widetilde{\psi}\rangle$, the three-level correlations are $\lambda_{123}=\left|\widetilde{\phi}_{1} \wedge \widetilde{\phi}_{2} \wedge \widetilde{\phi}_{3}\right|=0$, whereas $\lambda_{i j} \neq 0$, i.e., $|\widetilde{\psi}\rangle$ only contains two-level correlations.

The quantity $\lambda_{i_{1} \ldots i_{k}}$ is independent of the choice of hypo-states as long as $\operatorname{Sp}\left\{\left|\varphi_{i_{1}}\right\rangle, \ldots,\left|\varphi_{i_{k}}\right\rangle\right\} \cong$ $\mathcal{H}_{i_{1} \ldots i_{k}}$. To see this, let $\left\{\left|\varphi_{i_{l}}\right\rangle\right\}_{l=1}^{k}$ form an orthonormal basis of $\mathcal{H}_{i_{1} \ldots i_{k}}$ and define another set $\left\{\left|\varphi_{i_{l}}^{\prime}\right\rangle\right\}_{l=1}^{k}$ of arbitrary basis vectors via

$$
\left|\varphi_{i_{l}}^{\prime}\right\rangle=\sum_{m} c_{l m}\left|\varphi_{i_{m}}\right\rangle
$$

where $c_{l m}$ are elements of a complex-valued invertible $k \times k$ matrix. Define the corresponding set of re-states $\left\{\left|\phi_{i_{l}}^{\prime}\right\rangle\right\}_{l=1}^{k}$ as $\left|\phi_{i_{l}}^{\prime}\right\rangle=L_{\psi}\left|\varphi_{i_{l}}^{\prime}\right\rangle$. By the anti-linearity of the relative state map, we obtain

$$
\left|\phi_{i_{l}}^{\prime}\right\rangle=L_{\psi}\left(\sum_{m} c_{l m}\left|\varphi_{i_{m}}\right\rangle\right)=\sum_{m} c_{l m}^{*}\left|\phi_{i_{m}}\right\rangle .
$$

Explicit evaluation of the wedge product of the non-orthogonal basis elements yields

$$
\varphi_{i_{1}}^{\prime} \wedge \cdots \wedge \varphi_{i_{k}}^{\prime}=\left(\sum_{j_{1} \ldots j_{k}} \epsilon_{j_{1} \ldots j_{k}} c_{1_{1}} \cdots c_{k j_{k}}\right) \varphi_{i_{1}} \wedge \cdots \wedge \varphi_{i_{k}}
$$

where $\epsilon_{\nu_{1} \ldots \nu_{d}}$ denotes the Levi-Civita tensor. By performing the corresponding expansion of 
the set of primed re-states $\phi_{\mu}^{\prime}$, we obtain

$$
\phi_{i_{1}}^{\prime} \wedge \cdots \wedge \phi_{i_{k}}^{\prime}=\left(\sum_{j_{1} \ldots j_{k}} \epsilon_{j_{1} \ldots j_{k}} c_{1 j_{1}}^{*} \cdots c_{k j_{k}}^{*}\right) \phi_{i_{1}} \wedge \cdots \wedge \phi_{i_{k}},
$$

which is essentially the same expression as in Eq. (201) up to a complex conjugation of the coefficient $c_{l m}$. Thus, we conclude that

$$
\left|\phi_{i_{1}} \wedge \cdots \wedge \phi_{i_{k}}\right|=\frac{\left|\phi_{i_{1}}^{\prime} \wedge \cdots \wedge \phi_{i_{k}}^{\prime}\right|}{\left|\varphi_{i_{1}}^{\prime} \wedge \cdots \wedge \varphi_{i_{k}}^{\prime}\right|} .
$$

To simplify the notation, we henceforth assume that a set of hypo-states form an orthogonal basis and thus omit the denominator in Eq. (17).

The correlation quantities $\lambda_{i_{1} \ldots i_{k}}$ have the following properties. If the re-states $\left(\phi_{i_{1}}, \ldots, \phi_{i_{k}}\right)$ are linearly dependent, then $\lambda_{i_{1} \ldots i_{k}}=0$, which reflects that one can find two hypo-states in $\mathcal{H}_{i_{1} \ldots i_{k}}$ that maps to the same ray in $\mathcal{H}_{B}$. Furthermore, $\lambda_{i_{1} \ldots i_{k}}$ vanishes if $|\psi\rangle$ lacks support in some part of the subspace spanned by the hypo-states (one or several re-states will have zero norm). On the other hand, $\max \lambda_{i_{1} \ldots i_{k}}=(1 / \sqrt{k})^{k}$ and this value is saturated if $|\psi\rangle$ is maximally entangled on $\mathcal{H}_{i_{1} \ldots i_{k}}$ and $\left|\varphi_{i}\right\rangle, i \in\left(i_{1} \ldots i_{k}\right)$ span this subspace.

The quantities $\lambda_{i_{1} \ldots i_{k}}$ are in general not invariant under local unitaries. To see this, consider the local unitary transformation $|\psi\rangle \mapsto\left|\psi^{\prime}\right\rangle=U_{A} \otimes \hat{1}_{B}|\psi\rangle$, which implies that $\left|\phi_{i}\right\rangle \mapsto\left|\phi_{i}^{\prime}\right\rangle=\left\langle\varphi_{i}\left|U_{A} \otimes \hat{1}_{B}\right| \psi\right\rangle$. In other words, the transformed re-states would correspond to a set of hypo-states $\left|\varphi_{i}^{\prime}\right\rangle=U_{A}^{\dagger}\left|\varphi_{i}\right\rangle$, defining a different subspace decomposition of $\mathcal{H}_{A}$ leading to that $\lambda_{i_{1} \ldots i_{k}}$ may change. (The exception is $\lambda_{d}=\phi_{1} \wedge \cdots \wedge \phi_{d}$ that contains all re-states.) However, we have seen that the different choices of orthonormal bases of hypo-states are equivalent with local unitary transformations (on subsystem A) of the global state, and hence the question of invariance under change of hypo-states are equivalent to that of invariance under local unitary transformations. We now define

$$
\Lambda_{k}^{2}=d^{k}\left(\begin{array}{l}
d \\
k
\end{array}\right)^{-1} \sum_{i_{1}<\ldots<i_{k}}^{d} \lambda_{i_{1} \ldots i_{k}}^{2},
$$

where the sum is over all unique $k$-tuples of re-states and the normalization factor on the right-hand side is chosen so that $\Lambda_{k}=1$ for all $k$ if the global state is maximally entangled.

Theorem. For a $d \times d$-dimensional bipartite system, the members of the set $\left\{\Lambda_{k}\right\}_{k=1}^{d}$ are invariant under local unitary transformations.

Proof. Let $|\psi\rangle=\sum_{i=1}^{d} \sqrt{p_{i}}|i i\rangle$ be the bipartite state on Schmidt form. We can make a choice of hypo-states such that $\left|\varphi_{i}\right\rangle=|i\rangle$, with the corresponding re-states $\left|\phi_{i}\right\rangle=L_{\psi}\left|\varphi_{i}\right\rangle=$ $\sqrt{p_{i}}|i\rangle$. First, we consider a local unitary on subsystem $B$, i.e.,

$$
|\psi\rangle \mapsto|\widetilde{\psi}\rangle=\hat{1}_{A} \otimes U_{B}|\psi\rangle=\sum_{i=1}^{d} \sqrt{p_{i}}|i\rangle \otimes U_{B}|i\rangle,
$$

from which we se that the re-states transform according to $\left|\phi_{i}\right\rangle \mapsto\left|\widetilde{\phi}_{i}\right\rangle=U_{B}\left|\phi_{i}\right\rangle$. The corresponding transformation of the $k$-vectors then reads

$$
\phi_{i_{1}} \wedge \cdots \wedge \phi_{i_{i}} \mapsto \widetilde{\phi}_{i_{1}} \wedge \cdots \wedge \widetilde{\phi}_{i_{k}}=U_{B}^{\otimes k}\left(\phi_{i_{1}} \wedge \cdots \wedge \phi_{i_{k}}\right),
$$


which means that the unitary $U_{B}$ on $\mathcal{H}_{B}$ induces a unitary $U_{B}^{\otimes k}$ on the exterior space $\Omega^{k}\left(\mathcal{H}_{B}\right)$. Clearly, this cannot change the norm of the $k$-vector, since

$$
\widetilde{\lambda}_{i_{1} \ldots i_{k}}^{2}=\left(\phi_{i_{1}} \wedge \cdots \wedge \phi_{i_{k}}\right)^{\dagger}\left(U_{B}^{\otimes k}\right)^{\dagger} U_{B}^{\otimes k}\left(\phi_{i_{1}} \wedge \cdots \wedge \phi_{i_{k}}\right)=\left|\phi_{i_{1}} \wedge \cdots \wedge \phi_{i_{k}}\right|^{2}=\lambda_{i_{1} \ldots i_{k}}^{2},
$$

and thus we have that $\widetilde{\Lambda}_{k}=\Lambda_{k}$ under $U_{B}$.

Consider now a local unitary on subsystem $A$, i.e., let $|\psi\rangle$ be defined as before but let $|\psi\rangle \mapsto|\widetilde{\psi}\rangle=U_{A} \otimes \hat{1}_{B}|\psi\rangle$. In this case, the re-states transform as

$$
\left|\phi_{i}\right\rangle \mapsto\left|\widetilde{\phi}_{i}\right\rangle=L_{\widetilde{\psi}}\left|\varphi_{i}\right\rangle=\left\langle\varphi_{i}\left|U_{A} \otimes \hat{1}_{B}\right| \psi\right\rangle=L_{\psi} U_{A}^{\dagger}\left|\varphi_{i}\right\rangle=L_{\psi}\left|\widetilde{\varphi}_{i}\right\rangle
$$

i.e, a local unitary on subsystem $A$ is equivalent to the inverse transformation of the hypostates. If we denote $\left(U_{A}^{\dagger}\right)_{i j}=u_{i j}$, the transformed hypo-states are related to the original ones according to $\left|\widetilde{\varphi}_{j}\right\rangle=\sum_{i} u_{i j}\left|\varphi_{i}\right\rangle$, and we have that $\left|\widetilde{\phi}_{j}\right\rangle=L_{\psi}\left|\widetilde{\varphi}_{j}\right\rangle=\sum_{i} u_{i j}^{*} L_{\psi}\left|\varphi_{i}\right\rangle=\sum_{i} u_{i j}^{*}\left|\phi_{i}\right\rangle$. To show that $\widetilde{\Lambda}_{k}=\Lambda_{k}$, we first note that the $U_{A}$ induces a corresponding transformation of the $k$-vectors

$$
\phi_{j_{1}} \wedge \cdots \wedge \phi_{j_{k}} \mapsto \widetilde{\phi}_{j_{1}} \wedge \cdots \wedge \widetilde{\phi}_{j_{k}}=\left(\sum_{i_{1}} u_{i_{1} j_{1}}^{*} \phi_{i_{1}}\right) \wedge \cdots \wedge\left(\sum_{i_{k}} u_{i_{k} j_{k}}^{*} \phi_{i_{k}}\right),
$$

and summing the squared norms of the new $k$-vectors, we get

$$
\sum_{\substack { 1 \leq j_{1}<\ldots<j_{k} \leq d \\
\begin{subarray}{c}{1 \leq j_{1}<\ldots<j_{k} \leq d \\
1 \leq \mu_{1}<\ldots<\mu_{k} \leq d \\
i_{1} \ldots i_{k} \\
m_{1} \ldots m_{k}{ 1 \leq j _ { 1 } < \ldots < j _ { k } \leq d \\
\begin{subarray} { c } { 1 \leq j _ { 1 } < \ldots < j _ { k } \leq d \\
1 \leq \mu _ { 1 } < \ldots < \mu _ { k } \leq d \\
i _ { 1 } \ldots i _ { k } \\
m _ { 1 } \ldots m _ { k } } }\end{subarray}}\left|\widetilde{\phi}_{j_{1}} \wedge \cdots \wedge \widetilde{\phi}_{j_{k}}\right|^{2}=
$$

Here, we have used that the set $\left\{\phi_{\mu_{1}} \wedge \cdots \wedge \phi_{\mu_{k}}\right\}_{1 \leq \mu_{1}<\ldots<\mu_{k} \leq d}$ is an orthogonal basis of $\Omega^{k}\left(\mathcal{H}_{B}\right)$, which follows from the orthogonality of the Schmidt-basis and that $\left|\phi_{i}\right\rangle=\sqrt{p_{i}}|i\rangle$. Now, to see that the factors labeled by $\mu_{1} \ldots \mu_{k}$ each sum up to one as required, first note that the rows of a unitary is an orthonormal set of vectors, i.e., we have that $\sum_{j} u_{\mu_{i j}}^{*} u_{\mu_{m} j}=\delta_{i m}$. The determinant of the identity can then be expanded according to

$$
\begin{aligned}
1=\operatorname{det} \delta_{i m} & =\frac{1}{k !} \sum_{\substack{j_{1} \ldots j_{k} \\
i_{1} \ldots i_{k} \\
m_{1} \ldots m_{k}}} \epsilon_{i_{1} \ldots i_{k}} \epsilon_{m_{1} \ldots m_{k}} u_{\mu_{i_{1}} j_{1}}^{*} u_{\mu_{m_{1}} j_{1}} \cdots u_{\mu_{i_{k}} j_{k}}^{*} u_{\mu_{m_{k}} j_{k}} \\
& =\sum_{\substack{1 \leq j_{1}<\ldots<j_{k} \leq d \\
i_{1} \ldots i_{k} \\
m_{1} \ldots m_{k}}} \epsilon_{i_{1} \ldots i_{k}}^{\mu_{1} \ldots \mu_{k}} \epsilon_{m_{1} \ldots m_{k}}^{\mu_{1} \ldots \mu_{k}} u_{i_{1} j_{1}}^{*} u_{m_{1} j_{1}} \cdots u_{i_{k} j_{k}}^{*} u_{m_{k} j_{k}},
\end{aligned}
$$

where we have used the definition of the Levi-Civita tensor and that we can restrict the sums over $j_{1} \ldots j_{k}$.

The $k=1$ invariant is just normalization and does not provide any information about the correlation between the subsystems. Therefore, we take the correlation measures to consist of the set $\left\{\Lambda_{k}\right\}_{k=2}^{d}$. 
Note that the $k$-level invariants are not independent since $\Lambda_{k} \neq 0$ implies that $\Lambda_{l} \neq 0$ for all $l<k$. Geometrically, this expresses the fact that a non-zero volume must be bounded by non-zero areas. More explicitly, the lower order invariants are related to $\Lambda_{d}$ as

$$
\Lambda_{k} \geq\left(\begin{array}{l}
d \\
k
\end{array}\right)^{1 / 2}\left(\Lambda_{d}\right)^{k / d}, d>k
$$

which gives a lower bound for $k$ th order invariant.

We may relate the $\Lambda_{k}$ 's to known entanglement measures by using the Schmidt form $|\psi\rangle=$ $\sum_{k=1}^{d} \sqrt{p_{k}}\left|\varphi_{k}\right\rangle \otimes\left|\phi_{k}\right\rangle$, where $\left\langle\varphi_{k} \mid \varphi_{l}\right\rangle=\delta_{k l}$ and $\left\langle\phi_{k} \mid \phi_{l}\right\rangle=\delta_{k l}$, such that $L_{\psi}\left|\varphi_{k}\right\rangle=\sqrt{p_{k}}\left|\phi_{k}\right\rangle$. Since the re-states are subnormalized, mutually orthogonal vectors, it follows that

$$
\Lambda_{k}^{2}=\sum_{i_{1}<\ldots<i_{k}}\left|\phi_{i_{1}} \wedge \cdots \wedge \phi_{i_{k}}\right|^{2}=\sum_{i_{1}<\ldots<i_{k}} p_{i_{1}} \cdots p_{i_{k}} .
$$

Hence, the invariants are equivalent to the symmetric polynomials in the Schmidt coefficients, i.e., the concurrence hierarchies proposed in Ref. [14. The pure state invariant $\Lambda_{2}$ is recognized as the $I$ concurrence $[15]$

$$
C_{I}^{2}=4 \sum_{i<j} p_{i} p_{j}
$$

up to a factor. For $d=2$ (qubit) systems, $\Lambda_{2}$ is the only non-trivial invariant and equals half the pure state concurrence [18.

The relative state approach may further be used to give the following alternative geometric interpretation of pure state concurrence for qubit systems. Let $|\Psi\rangle=\sqrt{p_{0}}|00\rangle+\sqrt{p_{1}}|11\rangle$ and consider the orthonormal hypo-states $\left|\varphi_{0}\right\rangle=\alpha|0\rangle+\beta|1\rangle$ and $\left|\varphi_{1}\right\rangle=-\beta^{*}|0\rangle+\alpha^{*}|1\rangle$ with complex-valued $\alpha$ and $\beta$ such that $|\alpha|^{2}+|\beta|^{2}=1$. The corresponding re-states read $\left|\phi_{0}\right\rangle=\sqrt{p_{0}} \alpha^{*}|0\rangle+\sqrt{p_{1}} \beta^{*}|1\rangle \sim \mathbf{a}=\left(\sqrt{p_{0}} \alpha^{*}, \sqrt{p_{1}} \beta^{*}\right)$ and $\left|\phi_{1}\right\rangle=-\sqrt{p_{0}} \beta|0\rangle+\sqrt{p_{1}} \alpha|1\rangle \sim \mathbf{b}=$ $\left(-\sqrt{p_{0}} \beta, \sqrt{p_{1}} \alpha\right)$. The area $\mathcal{A}$ spanned by $\mathbf{a}$ and $\mathbf{b}$ is

$$
\mathcal{A}=\sqrt{|\mathbf{a}|^{2}|\mathbf{b}|^{2}-\left|\mathbf{a}^{*} \cdot \mathbf{b}\right|^{2}}=\sqrt{p_{0} p_{1}},
$$

which is half the pure state concurrence of the two-qubit state $\psi$. Thus, concurrence is essentially the area spanned by two re-states, as is shown in Fig. 1

\subsection{Mixed state correlation measures}

Let $\varrho \in \mathcal{S}\left(\mathcal{H}_{A} \otimes \mathcal{H}_{B}\right)$ be a bipartite state and assume that $d=\operatorname{dim} \mathcal{H}_{A} \leq \operatorname{dim} \mathcal{H}_{B}$. Let $\left\{\tau_{i}\right\}_{i=1}^{d^{2}}, \tau_{i} \in \mathcal{B}\left(\mathcal{H}_{A}\right)$, be a set of Hermitean operators on $\mathcal{H}_{A}$ such that $\operatorname{Sp}\left\{\tau_{i}\right\} \cong \mathcal{B}\left(\mathcal{H}_{A}\right)$ and define the corresponding set of operators $\left\{\pi_{i}\right\}_{i=1}^{d^{2}}, \pi_{i} \in \mathcal{S}^{\prime}\left(\mathcal{H}_{B}\right)$, as

$$
\pi_{i}=\mathfrak{L}_{\varrho}\left(\tau_{i}\right)=\operatorname{Tr}_{A}\left[\tau_{i} \otimes \hat{1} \varrho\right] .
$$

The basic correlation measures now read

$$
v_{i_{1} \ldots i_{k}}=\frac{\left|\pi_{i_{1}} \wedge \cdots \wedge \pi_{i_{k}}\right|}{\left|\tau_{i_{1}} \wedge \cdots \wedge \tau_{i_{k}}\right|}
$$

In analogy with the pure state case, these measures are independent of choice of $\left\{\tau_{i}\right\}$. In particular, if $\left\{\tau_{i}\right\}$ is an orthogonal set the denominator in Eq. (36) ) can be omitted. Note, 
however, the operational interpretation of $\left\{\tau_{i}\right\}$ and $\left\{\pi_{i}\right\}$ as states cannot be maintained for such a choice, since the space of density operators cannot be equipped with a complete orthogonal basis of positive operators. Nonetheless, due to the independence of the choice of $\left\{\tau_{i}\right\}$, we refer to $\left\{\tau_{i}\right\}$ and $\left\{\pi_{i}\right\}$ as hypo-states and re-states in the following, regardless of whether all members of the sets represent valid states or not.

To evaluate the wedge product, it is convenient to move to the Hilbert-Schmidt representation of states and observables as real-valued vectors and matrices. Thus, we make the substitutions $\varrho \rightarrow M,\left\{\tau_{i}\right\} \rightarrow\left\{\mathbf{a}_{i}\right\}$, and $\left\{\pi_{i}\right\} \rightarrow\left\{\mathbf{b}_{i}\right\}$, where $\mathbf{a}_{i}$ and $\mathbf{b}_{i}$ are related via the linear map $\mathbf{a}_{i} \rightarrow \mathbf{b}_{i}=M^{\mathrm{T}} \mathbf{a}_{i}$. Then

$$
\pi_{i_{1}} \wedge \cdots \wedge \pi_{i_{k}} \rightarrow \mathbf{b}_{i_{1}} \wedge \cdots \wedge \mathbf{b}_{i_{k}} .
$$

To illustrate this substitution, let us consider the case of a product state $\varrho=\rho_{A} \otimes \rho_{B}$. We find $M=\mathbf{r}_{A} \mathbf{r}_{B}^{\mathrm{T}}$, where $r_{A ; i}=\operatorname{Tr}\left[K_{i}^{A} \rho_{A}\right]$ and $r_{B ; i}=\operatorname{Tr}\left[K_{i}^{B} \rho_{B}\right]$ for some local operator bases $\left\{K_{i}^{A}\right\}$ and $\left\{K_{i}^{B}\right\}$. Hence, for a product state, the relative state map takes any $\mathbf{a} \in \mathcal{V}_{A}$ to a vector proportional to $\mathbf{r}_{B}: M^{\mathrm{T}} \mathbf{a}=\mathbf{r}_{B}\left(\mathbf{r}_{A}^{\mathrm{T}} \cdot \mathbf{a}\right)$, which implies $\left|\left(M^{\mathrm{T}} \mathbf{a}_{1}\right) \wedge \cdots \wedge\left(M^{\mathrm{T}} \mathbf{a}_{k}\right)\right|=0$ for any $k$-tuple $\left(\mathbf{a}_{1}, \ldots, \mathbf{a}_{k}\right) \in \mathcal{V}_{A}$.

We now define the correlation measures

$$
\Upsilon_{k}^{2}=d^{2 k}\left(\begin{array}{c}
d^{2} \\
k
\end{array}\right)^{-1} \sum_{i_{1}<\ldots<i_{k}}^{d^{2}} v_{i_{1} \ldots i_{k}}^{2},
$$

where the normalization factor is chosen such that $\Upsilon_{k}=1$ for maximally entangled states.

To demonstrate that $\Upsilon_{k}$ are invariant under local unitary operations, we first need to define the corresponding transformation in the Hilbert-Schmidt representation. Let $\varrho$ be a state and let $\mathbf{r}$ be the Hilbert-Schmidt representation of the state given by $r_{i}=\operatorname{Tr}\left[K_{i} \varrho\right]$. Furthermore, define $\varrho^{\prime}=U \varrho U^{\dagger}$ where $U$ is an arbitrary unitary transformation. Then

$$
r_{i}^{\prime}=\operatorname{Tr}\left[K_{i} \varrho^{\prime}\right]=\operatorname{Tr}\left[U^{\dagger} K_{i} U \varrho\right],
$$

and thus the transformation of $\varrho$ corresponds to the inverse transformation of the basis elements $K_{i}^{\prime}=U^{\dagger} K_{i} U$. From the the orthonormality of $\left\{K_{i}\right\}$ we see that $\operatorname{Tr}\left[K_{i}^{\prime} K_{j}^{\prime}\right]=$ $\operatorname{Tr}\left[U^{\dagger} K_{i} U U^{\dagger} K_{j} U\right]=\delta_{i j}$, i.e., $\left\{K_{i}^{\prime}\right\}$ is also an orthonormal basis. The transformation of $\mathbf{r}$ is given by the orthogonal transformation

$$
\mathbf{r}^{\prime}=R \mathbf{r}, R_{i j}=\operatorname{Tr}\left[K_{i} K_{j}^{\prime}\right] .
$$

Since $U$ is continuously connected to the identity, $R$ is too, and hence the transformation is a rotation. The transformation $U$ is also trace-preserving, which implies that $R$ is restricted to act on a $d^{2}-1$ dimensional subspace of $\mathcal{V}$, namely the plane orthogonal to the identity vector $\mathbf{v}_{I}$ with elements $\left(\mathbf{v}_{I}\right)_{i}=\operatorname{Tr}\left[K_{i}\right]$.

A local unitary transformation of a bipartite state

$$
\varrho \mapsto \varrho^{\prime}=U_{A} \otimes U_{B} \varrho U_{A}^{\dagger} \otimes U_{B}^{\dagger}
$$

induces the transformation $M \mapsto M^{\prime}=R_{B} M R_{A}^{\mathrm{T}}$, where

$$
\left(R_{A}\right)_{i j}=\operatorname{Tr}\left[U_{A}^{\dagger} K_{i}^{A} U_{A} K_{j}^{A}\right],\left(R_{B}\right)_{k l}=\operatorname{Tr}\left[U_{B}^{\dagger} K_{k}^{B} U_{B} K_{l}^{B}\right] .
$$


To see that the $\Upsilon_{k}$ 's are invariant under such transformations, it suffices to note that the above proof of the invariance of the pure state quantities $\Lambda_{k}$ under unitary transformations, immediately goes through for local rotations of the real vectors $\mathbf{a}_{i}$ and $\mathbf{b}_{i}$ representing the hypo- and re-states, respectively.

For any bipartite $\varrho$, there exists a unique Schmidt form

$$
\varrho=\sum_{i} \kappa_{i} \widetilde{K}_{i}^{A} \otimes \widetilde{K}_{i}^{B}
$$

where the Hermitian $\left\{\widetilde{K}_{i}^{A}\right\}$ and $\left\{\widetilde{K}_{i}^{B}\right\}$ are the particular orthonormal bases of operators on $\mathcal{H}_{A}$ and $\mathcal{H}_{B}$ - corresponding to the pure state Schmidt-bases - and the real numbers $\left\{\kappa_{i}\right\}$ are singular values of $M_{k l}=\operatorname{Tr}\left(K_{k}^{A} \otimes K_{l}^{B} \varrho\right)$. In analogy with the pure state case, the invariants $\Upsilon_{k}^{2}$ can be seen to be equivalent to the symmetric polynomials in $\kappa_{i}^{2}$, a form of correlation measures similar to those proposed in Ref. [19].

Since classical communication can increase correlations, it follows that $\Upsilon_{k}$ may increase under LOCC. However, as the following theorem shows, $\Upsilon_{k}$ are non-increasing under local operations.

Theorem. Suppose $\Upsilon_{k} \mapsto \widetilde{\Upsilon}_{k}$ under a local operation

$$
\varrho \mapsto \widetilde{\varrho}=\mathcal{E}_{\mathrm{LO}}(\varrho)=\sum_{i j} A_{i} \otimes B_{j} \varrho A_{i}^{\dagger} \otimes B_{j}^{\dagger} .
$$

Then $\widetilde{\Upsilon}_{k} \leq \Upsilon_{k}$.

Proof. We first note that a local operation takes the form

$$
M \mapsto \widetilde{M}=S_{A} M S_{B}^{\mathrm{T}},
$$

and that the $S$ matrices have a polar decomposition $S=R|S|$, where $R$ is a rotation and $|S|=\sum_{i} q_{i} \mathbf{f}_{i} \mathbf{f}_{i}^{\mathrm{T}}, 0 \leq q_{i} \leq 1, \mathbf{f}_{i}^{\mathrm{T}} \cdot \mathbf{f}_{j}=\delta_{i j}$, is a positive matrix. Let us first consider the case where $\mathcal{E}_{\mathrm{LO}}=\mathcal{E}_{B}$ corresponding to $M \mapsto \widetilde{M}=M S_{B}^{\mathrm{T}}=M\left|S_{B}\right| R_{B}^{\mathrm{T}}$. Since we have already proved that $\Upsilon_{k}$ are invariant under local unitaries, we may absorb $R_{B}$ into the choice of hypo-states $\mathbf{b}_{i}=\sum_{j} b_{j}^{(i)} \mathbf{f}_{j}$. Thus, the action of $S_{B}$ becomes

$$
\mathbf{b}_{i} \mapsto \widetilde{\mathbf{b}}_{i}=S_{B} \mathbf{b}_{i}=\sum_{j} q_{j} b_{j}^{(i)} \mathbf{f}_{j}
$$

We further note that $\left|\mathbf{b}_{i_{1}} \wedge \cdots \wedge \mathbf{b}_{i_{k}}\right|^{2}$ is the norm of the vector $\mathbf{b}_{i_{1} \ldots i_{k}}=\mathbf{b}_{i_{1}} \wedge \cdots \wedge \mathbf{b}_{i_{k}}$ in the exterior space $\Omega^{k}(\mathcal{B})$ of $\mathcal{B}$. The set $\left\{\mathbf{f}_{\mu_{1}} \wedge \cdots \wedge \mathbf{f}_{\mu_{k}}\right\}_{1 \leq \mu_{1}<\ldots<\mu_{k} \leq d}$ is an orthonormal ordered basis of $\Omega^{k}(\mathcal{B})$. Thus,

$$
\mathbf{b}_{i_{1}} \wedge \cdots \wedge \mathbf{b}_{i_{k}}=\sum_{1 \leq \mu_{1}<\ldots<\mu_{k} \leq d} b_{\mu_{1} \ldots \mu_{k}}^{\left(i_{1} \ldots i_{k}\right)} \mathbf{f}_{\mu_{1}} \wedge \cdots \wedge \mathbf{f}_{\mu_{k}}
$$

where

$$
b_{\mu_{1} \ldots \mu_{k}}^{\left(i_{1} \ldots i_{k}\right)}=\sum_{m_{1} \ldots m_{k}} \epsilon_{m_{1} \ldots m_{k}}^{\mu_{1} \ldots \mu_{k}} b_{m_{1}}^{\left(i_{1}\right)} \cdots b_{m_{k}}^{\left(i_{k}\right)}
$$


and we may write

$$
v_{i_{1} \ldots i_{k}}^{2}=\left|\mathbf{b}_{i_{1}} \wedge \cdots \wedge \mathbf{b}_{i_{k}}\right|^{2}=\sum_{1 \leq \mu_{1}<\ldots<\mu_{k} \leq d}\left|b_{\mu_{1} \ldots \mu_{k}}^{\left(i_{1} \ldots i_{k}\right)}\right|^{2} .
$$

Now, under the local operation $\mathcal{E}_{B}$, the correlation measure transforms as $v_{i_{1} \ldots i_{k}}^{2} \mapsto \widetilde{v}_{i_{1} \ldots i_{k}}^{2}=$ $\left|\widetilde{\mathbf{b}}_{i_{1}} \wedge \cdots \wedge \widetilde{\mathbf{b}}_{i_{k}}\right|^{2}$, which can be written as

$$
\begin{aligned}
\widetilde{v}_{i_{1} \ldots i_{k}}^{2} & =\left|\left(\sum_{j_{1}} q_{j_{1}} b_{j_{1}}^{\left(i_{1}\right)} \mathbf{f}_{j_{1}}\right) \wedge \cdots \wedge\left(\sum_{j_{k}} q_{j_{k}} b_{j_{k}}^{\left(i_{k}\right)} \mathbf{f}_{j_{k}}\right)\right|^{2} \\
& =\sum_{1 \leq \mu_{1}<\ldots<\mu_{k} \leq d} q_{\mu_{1}}^{2} \cdots q_{\mu_{k}}^{2}\left|b_{\mu_{1} \ldots \mu_{k}}^{\left(i_{1} \ldots i_{k}\right)}\right|^{2} .
\end{aligned}
$$

Here, we have used that $q_{\mu}$ are independent of the indices $i_{1}, \ldots, i_{k}$. Since $0 \leq q_{\mu} \leq 1$, it follows that $v_{i_{1} \ldots i_{k}}$ is non-increasing. Thus, $\widetilde{\Upsilon}_{k} \leq \Upsilon_{k}$.

Finally, we need to consider the bi-local operation $M^{\prime}=S_{A} M S_{B}^{\mathrm{T}}$. This can be written as $M \mapsto M^{\prime}=R_{A}\left|S_{A}\right| M\left|S_{B}\right| R_{B}^{\mathrm{T}}$ and from the consecutive application of the above argument it is clear that

$$
\Upsilon_{k} \geq \widetilde{\Upsilon}_{k} \geq \Upsilon_{k}^{\prime}
$$

which completes the proof.

If we calculate the invariants $\left\{\Upsilon_{k}\right\}_{k=2}^{d^{2}}$ for a pure state, we expect them to contain redundant information, since the entanglement in the pure state is characterized by the set $\left\{\Lambda_{k}\right\}_{k=2}^{d}$ of pure state invariants. To see how this manifests, consider a pure state with the Schmidt form $|\psi\rangle=\sum_{i} \sqrt{p_{i}}|i i\rangle$. We make particular choice of the local basis operators, defining them in terms of the local Schmidt bases as

$$
\begin{aligned}
E_{k} & =|k\rangle\langle k|, k=1, \ldots, d, \\
F_{l l^{\prime}} & =\frac{1}{\sqrt{2}}\left(|l\rangle\left\langle l^{\prime}|+| l^{\prime}\right\rangle\langle l|\right), 1 \leq l<l^{\prime} \leq d, \\
G_{m m^{\prime}} & =\frac{i}{\sqrt{2}}\left(|m\rangle\left\langle m^{\prime}|-| m^{\prime}\right\rangle\langle m|\right), 1 \leq m<m^{\prime} \leq d .
\end{aligned}
$$

The Hilbert-Schmidt representation $M_{\psi}$ of the state $|\psi\rangle\langle\psi|$ is diagonal in this basis, with the diagonal values given by

$$
\begin{aligned}
e_{k}=p_{k} & =\operatorname{Tr}\left[E_{k}^{A} \otimes E_{k}^{B}|\psi\rangle\langle\psi|\right], \\
f_{l l^{\prime}}=\sqrt{p_{l} p_{l^{\prime}}} & =\operatorname{Tr}\left[F_{l l^{\prime}}^{A} \otimes F_{l l^{\prime}}^{B}|\psi\rangle\langle\psi|\right], \\
g_{m m^{\prime}}=-\sqrt{p_{m} p_{m^{\prime}}} & =\operatorname{Tr}\left[G_{m m^{\prime}}^{A} \otimes G_{m m^{\prime}}^{B}|\psi\rangle\langle\psi|\right] .
\end{aligned}
$$

Note that this is essentially the mixed state Schmidt decomposition given in Eq. (43) with mixed state Schmidt coefficients $\left\{e_{k}, f_{l l^{\prime}}, g_{m m^{\prime}}\right\}$, i.e., we have that

$$
|\psi\rangle\langle\psi|=\sum_{k=1}^{d} p_{k} E_{k}^{A} \otimes E_{k}^{B}+\sum_{1 \leq l<l^{\prime} \leq d} \sqrt{p_{l} p_{l^{\prime}}}\left(F_{l l^{\prime}}^{A} \otimes F_{l l^{\prime}}^{B}-G_{l l^{\prime}}^{A} \otimes G_{l l^{\prime}}^{B}\right) .
$$


The measures $\Upsilon_{k}$ are functions of the mixed state Schmidt coefficients $\left\{e_{k}, f_{l l^{\prime}}, g_{m m^{\prime}}\right\}$, which for pure states are, in turn, simple functions of the pure state Schmidt coefficients, as can be seen from Eq. (53). Hence $\Upsilon_{k}$ can be expressed in terms of the pure state measures $\Lambda_{k}$. For some $k$ this relation becomes simple, e.g., one can show that

$$
\Upsilon_{2}^{2}=2\left(\Lambda_{2}^{2}-\Lambda_{2}^{4}\right), \quad \Upsilon_{3}^{2}=2\left(\Lambda_{2}^{4}-\Lambda_{2}^{6}\right), \quad \Upsilon_{d^{2}}^{2}=\Lambda_{d}^{2 d}
$$

\section{Application: Quantum dynamics}

We illustrate the correlation measures $\Upsilon_{k}$ by looking at how the correlations of a maximally entangled state $|\psi\rangle=\frac{1}{\sqrt{d}} \sum_{i=1}^{d}|i i\rangle$ of a $d \times d$ dimensional system changes under two types of decoherence.

We first consider the depolarization channel $\mathcal{E}$ defined as the map

$$
\psi \mapsto \varrho_{W}=\mathcal{E}(\psi)=p \psi+(1-p) \varrho_{*}
$$

of $\psi$. Here, $\psi=|\psi\rangle\langle\psi|$ and $\varrho_{*}=\frac{1}{d^{2}} \hat{1}_{A B}$, i.e., the output $\varrho$ is a Werner state that connects the maximally entangled state $\psi$ for $p=1$ and the random mixture $\varrho_{*}$ for $p=0$. The relative state map $\mathfrak{L}_{W}$ induced by $\varrho$ acts on a hypo-state $\tau \in \mathcal{S}_{A}$ as

$$
\tau \mapsto \pi=\mathfrak{L}_{W}(\tau)=p \mathfrak{L}_{\psi}(\tau)+(1-p) \mathfrak{L}_{*}(\tau),
$$

where $\mathfrak{L}_{\psi}$ and $\mathfrak{L}_{*}$ are the maps induced by $\psi$ and $\varrho_{*}$, respectively. In particular, for any $Y \in \mathcal{B}\left(\mathcal{H}_{A}\right)$, we find $\mathfrak{L}_{*}(Y)=\frac{1}{d^{2}} \operatorname{Tr}[Y] \hat{1}_{B}$, i.e., the relative state map defined by $\varrho_{*}$ maps any element of $\mathcal{B}\left(\mathcal{H}_{A}\right)$ to an operator proportional to the reduced state of subsystem $B$.

Now, let $\left\{K_{i}^{A}\right\}$ and $\left\{K_{i}^{B}\right\}$ be orthonormal bases of $\mathcal{B}\left(\mathcal{H}_{A}\right)$ and $\mathcal{B}\left(\mathcal{H}_{B}\right)$, respectively, with the additional property that $K_{1}^{A}=\frac{1}{\sqrt{d}} \hat{1}_{A}$ and $K_{1}^{B}=\frac{1}{\sqrt{d}} \hat{1}_{B}$. This implies $\operatorname{Tr}\left[K_{i}^{A}\right]=$ $\operatorname{Tr}\left[K_{i}^{B}\right]=0$ for $i>1$. By choosing $\tau_{i}=K_{i}^{A}$, we obtain the re-states

$$
\begin{aligned}
& \pi_{1}=\mathfrak{L}_{W}\left(\tau_{1}\right)=\left(\frac{1}{d}\right)^{\frac{3}{2}} \hat{1}_{B}, \\
& \pi_{i}=\mathfrak{L}_{W}\left(\tau_{i}\right)=p \mathfrak{L}_{\psi}\left(\tau_{i}\right)=p \pi_{i}^{\prime}, i>1 .
\end{aligned}
$$

Let us use these expressions to evaluate first $\Upsilon_{2}^{2}$ explicitly. The individual terms are given by $v_{i_{1} i_{2}}=\left|\pi_{i_{1}} \wedge \pi_{i_{2}}\right|$, which can take two values

$$
\begin{aligned}
& v_{1 i_{2}}=\left|\pi_{1} \wedge \pi_{i_{2}}\right|=\frac{p}{d^{2}}, 1<i_{2}, \\
& v_{i_{1} i_{2}}=\left|\pi_{i_{1}} \wedge \pi_{i_{2}}\right|=\frac{p^{2}}{d^{2}}, 1<i_{1}<i_{2},
\end{aligned}
$$

where we have used that $\left|\pi_{i}^{\prime} \wedge \pi_{j}^{\prime}\right|=1 / d^{2}$ for all $i \neq j$. Hence, we have that

$$
\Upsilon_{2}^{2}=\left(\begin{array}{c}
d^{2} \\
k
\end{array}\right)^{-1} p^{2}\left(d^{2}-1+\left(\begin{array}{c}
d^{2}-1 \\
2
\end{array}\right) p^{2}\right)=\frac{p^{2}}{d^{2}}\left(2+\left(d^{2}-2\right) p^{2}\right) .
$$

Generalizing to arbitrary $k=2, \ldots, d^{2}$ yields

$$
\Upsilon_{k}^{2}=d^{2 k}\left(\begin{array}{c}
d^{2} \\
k
\end{array}\right)^{-1} \sum_{1 \leq i_{1}<\ldots<i_{k} \leq d^{2}} v_{i_{1} \ldots i_{k}}^{2}=p^{2(k-1)}\left[\frac{k}{d^{2}}+\left(1-\frac{k}{d^{2}}\right) p^{2}\right]
$$




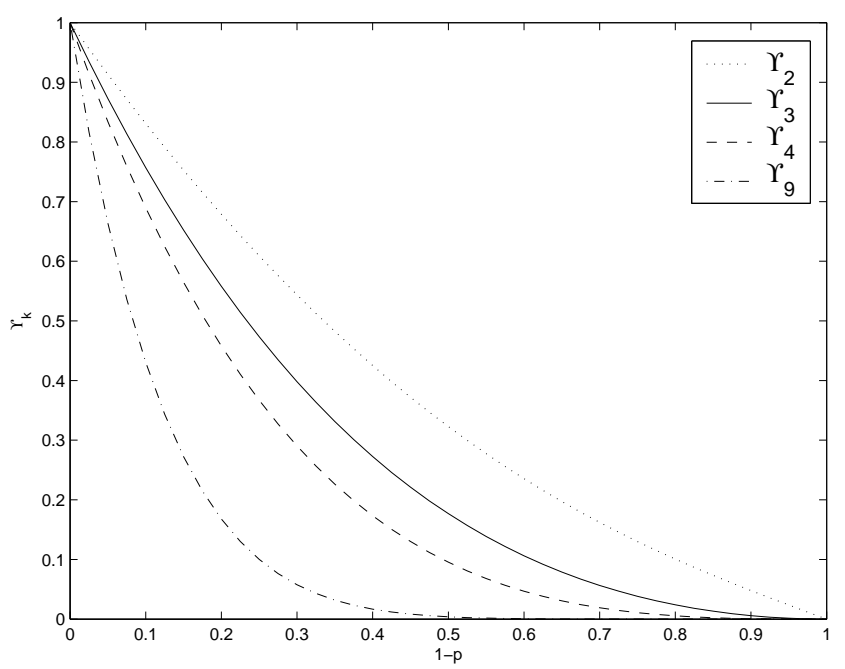

Fig. 3. The invariants of a maximally entangled $3 \times 3$ state undergoing depolarization. With increasing noise, all $\Upsilon_{k}$ tend to zero.

which vanishes when $p \rightarrow 0$. The $k=2,3,4,9$ invariants are shown in Fig. 3 for $d=3$.

Secondly, we look at how the invariants change under product-basis decoherence of the maximally entangled state $\psi$. Let the product basis be composed of the local Schmidt-bases, i.e., $E_{i}^{A} \otimes E_{j}^{B}=|i\rangle\langle i|\otimes| j\rangle\langle j|$. The channel $\mathcal{F}$ can be represented as

$$
\psi \mapsto \varrho_{D}=\mathcal{F}(\psi)=p \psi+(1-p) \sum_{i j=1}^{d} E_{i}^{A} \otimes E_{j}^{B} \psi E_{i}^{A} \otimes E_{j}^{B} .
$$

If we define the maximally decohered state $\Xi=\sum_{i j} E_{i}^{A} \otimes E_{j}^{B} \psi E_{i}^{A} \otimes E_{j}^{B}=\frac{1}{d} \sum_{i} E_{i}^{A} \otimes E_{i}^{B}$, then

$$
\mathfrak{L}_{D}\left(\tau_{i}\right)=p \mathfrak{L}_{\psi}\left(\tau_{i}\right)+(1-p) \mathfrak{L}_{\Xi}\left(\tau_{i}\right)
$$

We choose the local basis operators given in Eq.(52). With the identification $\left\{\tau_{i}\right\}=\left\{E_{k}, F_{l l^{\prime}}, G_{m m^{\prime}}\right\}$, we have $\mathfrak{L}_{\psi}\left(\tau_{i}\right)=\mathfrak{L}_{\Xi}\left(\tau_{i}\right)$ for $1 \leq i \leq d$, and $\mathfrak{L}_{\Xi}\left(\tau_{i}\right)=0$ for $i>d$. $\mathfrak{L}_{D}\left(\tau_{i}\right)$ takes two values in terms of $\mathfrak{L}_{\psi}\left(\tau_{i}\right)$ :

$$
\mathfrak{L}_{D}\left(\tau_{i}\right)=\left\{\begin{array}{ll}
\mathfrak{L}_{\psi}\left(\tau_{i}\right), & 1 \leq i \leq d \\
p \mathfrak{L}_{\psi}\left(\tau_{i}\right), & d<i \leq d^{2}
\end{array} .\right.
$$

It is then a matter of combinatorics to show that the invariants for general $k$ are given by

$$
\Upsilon_{k}^{2}=\left(\begin{array}{c}
d^{2} \\
k
\end{array}\right)^{-1} \sum_{l=0}^{k}\left(\begin{array}{c}
d \\
k-l
\end{array}\right)\left(\begin{array}{c}
d^{2}-d \\
l
\end{array}\right) p^{2 l} .
$$

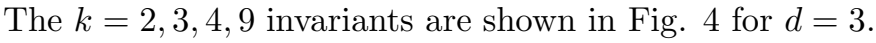

For the depolarization channel, all $\Upsilon_{k} \rightarrow 0$ when $p \rightarrow 0$, i.e., all $k$-level correlations are suppressed and vanish for the final product state. However, the Werner state for $p \neq 0$ inherits 


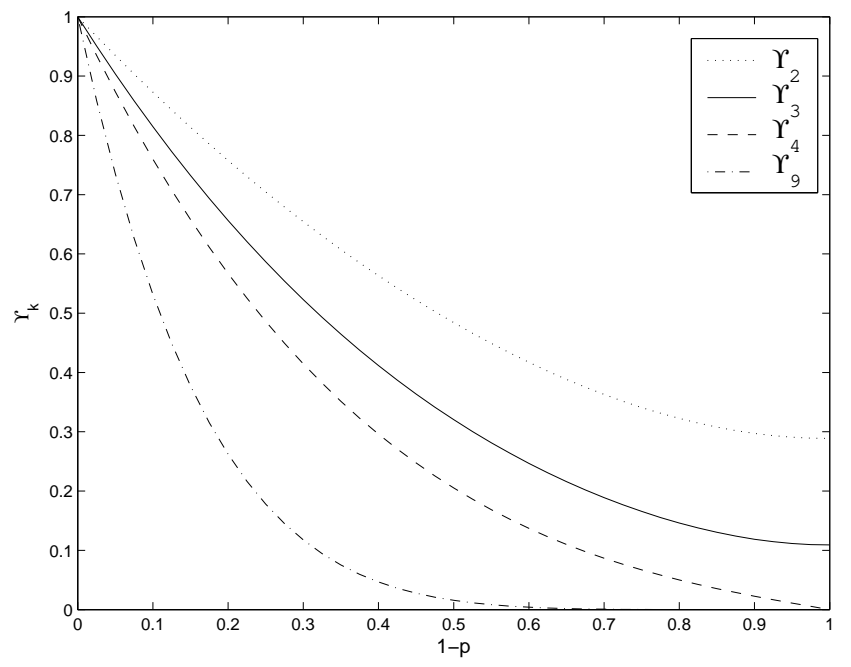

Fig. 4. The product basis decoherence of a maximally entangled state. Note that $\Upsilon_{k} \rightarrow 0$ when $p \rightarrow 0$ for $k>d$, while it goes to a finite value for $k \leq d$. The final state defined by $\mathcal{F}$ for $p=0$ is a maximally correlated separable state.

the symmetry of the maximally entangled state: for any choice of orthonormal measurement basis $\left\{\left|a_{i}\right\rangle\right\}$ (i.e., an observable) at site $A$, there is a corresponding orthonormal basis $\left\{\left|b_{i}\right\rangle\right\}$ at site $B$, in which the correlations (as measured by, e.g., mutual information) exhibited by the resulting probability distribution will be non-zero. In terms of the invariants, this is due to that $\Upsilon_{d^{2}} \neq 0$, or, in terms of the $k$-vectors, that for any choice $\left\{\left|a_{i}\right\rangle\right\}$ the objects $\mathfrak{L}_{W}\left(\left|a_{1}\right\rangle\left\langle a_{1}\right|\right) \wedge \cdots \wedge \mathfrak{L}_{W}\left(\left|a_{d}\right\rangle\left\langle a_{d}\right|\right)$ and $\mathfrak{L}_{W}\left(\tau_{1}\right) \wedge \cdots \wedge \mathfrak{L}_{W}\left(\tau_{d^{2}}\right)$ have a $d$-dimensional intersection.

For the product-basis decoherence channel, which is of interest as, e.g., a model for measurement einselection, we see that $\Upsilon_{k} \rightarrow 0$ for $k>d$ and $\Upsilon_{k} \rightarrow\left(\begin{array}{c}d^{2} \\ k\end{array}\right)^{-1 / 2}\left(\begin{array}{l}d \\ k\end{array}\right)^{1 / 2}$ for $k \leq d$, when $p \rightarrow 0$ (see Fig. 4). This can be related to proposed measures of classical correlations in quantum states [20, 21], in particular, quantum discord [21] defined as

$$
D\left(A: B \mid\left\{\tau_{i}\right\}\right)=S\left(\rho_{B}\right)-S(\rho)+\sum_{i} p_{i} S\left(\pi_{i} / p_{i}\right)
$$

where $p_{i}=\operatorname{Tr}\left[\pi_{i}\right], S(\rho)$ denotes the von Neumann entropy of the state $\rho$, and we have the restriction $\tau_{i} \in \mathcal{S}\left(\mathcal{H}_{A}\right)$, which ensures that $\mathfrak{L}_{\varrho}\left(\tau_{i}\right)=\pi_{i} \in \mathcal{S}^{\prime}\left(\mathcal{H}_{B}\right)$ and that $\pi_{i} / p_{i} \in \mathcal{S}\left(\mathcal{H}_{B}\right)$. This definition also utilizes a relative state construction in that they are derived from entropies over subsystem $B$ that are conditioned on measurements on subsystem $A$. The minimum discord of a state

$$
D_{\min }(A: B)=D\left(A: B \mid\left\{\tau_{i}\right\}_{\min }\right)=S\left(\rho_{B}\right)-S(\rho)+\min _{\left\{\tau_{i}\right\}}\left(\sum_{i} p_{i} S\left(\pi_{i} / p_{i}\right)\right)
$$

quantifies the amount of information lost in the optimal correlation measurement.

The final state $\Xi$ is the maximally correlated separable state and likewise the maximally correlated zero-discord state. A zero-discord state is characterized by that $D_{\min }=0$, which 
a)

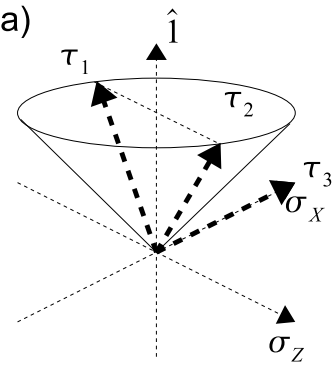

b)

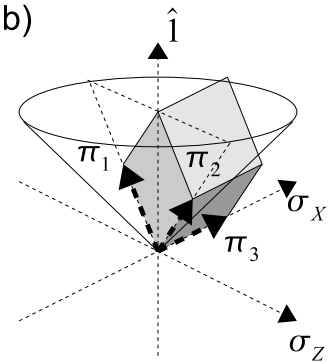

c)

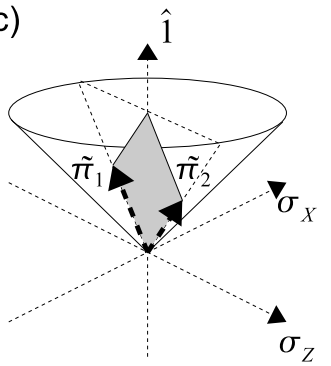

Fig. 5. An illustration of the mixed state relative states and the mixed state correlation measures for the $2 \otimes 2$ system. In a) we show a three dimensional subspace of $\mathcal{B}\left(\mathcal{H}_{A}\right)$, spanned by basis operators $K_{1}^{A}=\hat{1} / \sqrt{2}, K_{2}^{A}=\sigma_{x} / \sqrt{2}$ and $K_{3}^{A}=\sigma_{z} / \sqrt{2}$, and b) and c) shows the corresponding subspace of $\mathcal{B}\left(\mathcal{H}_{B}\right)$. The disc enclosed by the circle orthogonal to $\hat{1}$ is a subspace of the state space $\mathcal{S}$, i.e., the $x z$-plane of the Bloch-sphere, with the pure states on the boundary. The corresponding subspace of subnormalized states $\mathcal{S}^{\prime}$ is the cone with the circle as its base. Three (out of four) hypo-states $\tau_{1}=|0\rangle\left\langle 0\left|, \tau_{2}=\right| 1\right\rangle\langle 1|, \tau_{3}=K_{2}$ are shown in a). The re-states $\pi_{i}=\mathfrak{L}_{\psi}\left(\tau_{i}\right)$, defined by the maximally entangled state $\psi=\frac{1}{2} \sum_{k, l=0}^{1}|k k\rangle\langle l l|$, is shown in b), where the volume spanned is $v_{123}=\left|\pi_{1} \wedge \pi_{2} \wedge \pi_{3}\right|=(1 / 2)^{3}$. In c) the restates $\tilde{\pi}_{i}=\mathfrak{L}_{\Xi}\left(\tau_{i}\right)$ of the maximally classically correlated state $\Xi=\frac{1}{2}(|00\rangle\langle 00|+| 11\rangle\langle 11|)$, is shown, where $v_{123}=\left|\widetilde{\pi}_{1} \wedge \widetilde{\pi}_{2} \wedge \widetilde{\pi}_{3}\right|=0$ since $\widetilde{\pi}_{3}=0$. The quantity $v_{12}=1 / 4$ is the only nonzero contribution to the invariants, and hence $\Upsilon_{4}=\Upsilon_{3}=0$ and only $\Upsilon_{2} \neq 0$, which characterizes a two-qubit 0-discord state.

means that all its correlations can be extracted by a single measurement setup, and consequently that the state is robust under this particular measurement, and that the classical mutual information over the probability distribution obtained by measuring in a product basis equals the quantum mutual information of the state. This can be related to the invariants in the following way: If $\Upsilon_{k} \neq 0$ for $k>d$, then $D_{\min }(A: B) \neq 0$, and if $D_{\min }(A: B)=0$, then $\Upsilon_{k}=0$ for $k>d$. The first implication we understand as that the re-states span a $k>d$-dimensional subspace of $\mathcal{S}_{B}$, while the (complete) set of projectors constituting a measurement basis only span a $d$-dimensional subspace, thus the $k$-volume spanned by the re-states "collapses" into a $d$-volume upon measurement, which is what we see in the example of product basis decoherence. The restates of the pre- and post-measurement states $\psi$ and $\Xi$ are shown in Figs. $5 \mathrm{~b}$ and 5 c, respectively. Conversely, the second implication illustrates that the re-states of a zero-discord state, which is robust under some product basis measurement, can maximally span a $d$-dimensional subspace. It also follows that, contrary to the symmetric Werner state, that one can find a set of $d$-hypo-states $\left\{\left|a_{i}^{\prime}\right\rangle\right\}$ (bases $\left\{\left|a_{i}^{\prime}\right\rangle\right\}$ and $\left\{E_{i}\right\}$ are mutually unbiased) such that $\mathfrak{L}_{\Xi}\left(\left|a_{i}^{\prime}\right\rangle\left\langle a_{i}^{\prime}\right|\right)=\mathfrak{L}_{\Xi}\left(\left|a_{j}^{\prime}\right\rangle\left\langle a_{j}^{\prime}\right|\right)$, and none of the state's correlations can be extracted.

\section{Conclusions}

The concept of relative state, originally developed by Everett [1] to deal with the measurement problem in quantum mechanics, has been used to construct measures of correlations in pure bipartite quantum states of arbitrary dimension. The basic idea is to quantify how much information one observer can obtain about measurements that can be performed by another observer, if they are allowed only to do local, projective measurements. These correlation measures have been shown in detail to be invariant under local unitary transformations of 
the shared bipartite state. We have further shown that the present correlation measures coincide with those given by concurrence hierarchies [14] and $I$ concurrence [15, providing an alternative operational interpretation of these measures.

We have extended the notion of relative state to generalized measurements. This allows for studies of the correlation structure of mixed bipartite states. The corresponding measures quantify the total correlation in the sense that they vanish for product states, and are nonincreasing under local operations, but may increase under LOCC. We have illustrated the behavior of the mixed state correlation measures for bipartite systems of arbitrary dimension undergoing two different types of open system dynamics.

\section{Acknowledgments}

PR acknowledges financial support from the Göran Gustafsson Foundation. ES acknowledges support from the National Research Foundation and the Ministry of Education (Singapore).

1. A. Einstein, B. Podolsky, and N. Rosen (1935), Can quantum-mechanical description of physical reality be considered complete?, Phys. Rev., 47, 777-780.

2. E. Schrödinger (1935), Discussion of probability relations between separated systems, Proc. Cambridge Philos. Soc., 31, 555-563.

3. J. S. Bell (1966), On the problem of hidden variables in quantum mechanics, Rev. Mod. Phys., 38, 447-452.

4. M. Pawłowski, T. Paterek, D. Kaszlikowski, V. Scarani, A. Winter, and M. Żukowski (2008), Information causality as a physical principle, Nature, 461, 1101-1104.

5. A. K. Ekert (1991), Quantum cryptography based on Bells theorem, Phys. Rev. Lett., 67, 661-663.

6. C. H. Bennett, G. Brassard, C. Crépeau, R. Jozsa, A. Peres, and W. K. Wootters (1993), Teleporting an unknown quantum state via dual classical and Einstein-Podolsky-Rosen channels, Phys. Rev. Lett., 70, 1895-1899.

7. J. S. Bell (1965), On the Einstein-Podolsky-Rosen paradox, Physics, 1, 195.

8. J. F. Clauser, M. A. Horne, A. Shimony, and R. A. Holt (1969), Proposed experiment to test local hidden-variable theories, Phys. Rev. Lett., 23, 880-884.

9. C. H. Bennett, D. P. DiVincenzo, J. A. Smolin, and W. K. Wootters (1996), Mixed-state entanglement and quantum error correction, Phys. Rev. A, 54, 3824-3851.

10. C. H. Bennett, G. Brassard, S. Popescu, B. Schumacher, J. A. Smolin, and W. K. Wootters (1996), Purification of noisy entanglement and faithful teleportation via noisy channels, Phys. Rev. Lett., $76,722-725$.

11. H. Everett (1957), Relative state formulation of quantum mechanics, Rev. Mod. Phys., 29, 454-462.

12. R. Arens and V. S. Varadarajan (2000), On the concept of Einstein-Podolosky-Rosen states and their structure, J. Math. Phys., 41, 638-651.

13. H. M. Wiseman, S. J. Jones, and A. C. Doherty (2007), Steering, Entanglement, Nonlocality, and the Einstein-Podolsky-Rosen Paradox, Phys. Rev. Lett., 98, 140402.

14. H. Fan, K. Matsumoto, and H. Imai (2003), Quantify entanglement by concurrence hierarchy, J. Phys. A: Math. Gen., 36, 4151-4158.

15. P. Rungta, V. Bužek, C. M. Caves, M. Hillery, and G. J. Milburn (2001), Universal state inversion and concurrence in arbitrary dimensions, Phys. Rev. A, 64, 042315.

16. Z. Kurucz, M. Koniorczyk, and J. Janszky (2001), Teleportation with partially entangled states, Fortschr. Phys., 49, 1019-1025.

17. Z. Kurucz, M. Koniorczyk, P. Adam, and J. Janszky (2003), An operator description of entanglement matching in quantum teleportation, J. Opt. B: Quantum Semiclass. Opt., 5, S627-S632.

18. W. K. Wootters (1998), Entanglement of formation of an arbitrary state of two qubits, Phys. Rev. Lett., 80, 2245-2248.

19. P. Aniello and C. Lupo (2009), On the relation between Schmidt coefficients and entanglement, Open Sys. Information Dyn., 16, 127-143. 
20. L. Henderson and V. Vedral (2001), Classical, quantum and total correlations, J. Phys. A: Math. Gen., 34, 6899-6905.

21. H. Ollivier, and W. H. Zurek (2001), Quantum discord: A measure of the quantumness of correlations, Phys. Rev. Lett., 88, 017901. 